\title{
Article \\ Statistical analysis of dynamic subgrid modeling approaches in large eddy simulation
}

\author{
Mohammad Khalid Hossen ${ }^{1}$, Asokan Variyath ${ }^{1}$ and Jahrul Alam ${ }^{1 *(D)}$ \\ 1 Department of Mathematics and Statistics, Memorial University of Newfoundland 1; alamj@mun.ca \\ * Correspondence: alamj@mun.ca; Tel.: +1-709-864-8071 (J.A.)
}

\begin{abstract}
In large eddy simulation (LES) of turbulent flows, the most critical dynamical processes to be considered by dynamic subgrid models to account for an average cascade of kinetic energy from the largest to the smallest scales of the flow is not fully clear. Furthermore, evidence of vortex stretching being the primary mechanism of the cascade is not out of the question. In this article, we study some essential statistical characteristics of vortex stretching and its role in dynamic approaches of modeling subgrid-scale turbulence. We have compared the interaction of subgrid stresses with the filtered quantities among four models using invariants of the velocity gradient tensor. This technique is a single unified approach to studying a wide range of length scales in the turbulent flow. In addition, it also provides a rational basis for the statistical characteristics a subgrid model must serve in physical space to ensure an appropriate cascade of kinetic energy. Results indicate that the stretching mechanism extracts energy from the large-scale straining motion and passes it onto small-scale stretched vortices.
\end{abstract}

Keywords: keyword 1; keyword 2; keyword 3 (List three to ten pertinent keywords specific to the article; yet reasonably common within the subject discipline.)

\section{Introduction}

Turbulence is a high-dimensional chaotic system [1-3]. To correctly capture the breakdown of eddies through a hierarchical process of energy cascade in turbulent flows, the direct numerical simulation (DNS) approach must deal with an enormous number of degrees of freedom [4]. In contrast, the LES technique captures the statistics of energycontaining eddies [5]. A subgrid-scale model [6] often represents the residual stress $\left(\tau_{i j}\right)$ exerted by the discarded small-scale eddies. It is also worth mentioning that in complex flows around solid geometries, the predicted statistics of momentum and energy transport achieve a high level of accuracy in LES, albeit using a DNS-like cost $[7,8]$. Thus, a comprehensive research goal in LES is to find the best cost-effective strategy that can predict turbulence dynamics accurately in a cost effective manner [9].

In this article, we demonstrate some causal connections between the vortex stretching mechanism and the local energy flux from large to small scales. For instance, consider a rectilinear vortex tube that has the largest velocity components in the plane orthogonal to its axis. The vorticity and the strain are in complementary distribution for such a vortex tube, i.e. vorticity is zero outside the tube and strain is zero inside [10]. Such tubes tend to induce a turbulent stress, which indicates that stretching of vortex tubes will extract energy from surrounding large-scale strain. Based on the equations representing the evolution of strain and enstrophy [11,12], however, the role of vortex tubes observed in actual turbulent flows is of considerable depate. There is thus a need of further investigations in this exciting direction.

This article presents an eddy-viscosity model that is based on the second invariant of the velocity gradient tensor as well as the vortex stretching vector. If the energy flux from large- to small-scales is caused by vortex stretching, this model will yield the same 
turbulence statistics predicted by other dynamic subgrid models [3,13]. Our study here is one way to confirm a causal connection between vortex stretching and energy cascade. Another reason of considering vortex stretching to represent subgrid dissipation is that the vorticity field is more intermittent than the strain field [14-17]. The intermittency of turbulence is a result from the subtle balance between convective transport and diffusive dissipation. In this regard, the transport of five invariants of the velocity gradient tensor through the "restricted Euler equations" is important for understanding the transport of energy flux in turbulent flows [2,15,16]. In previous studies [17-19], we demonstrate that these invariants play an essential role in modeling wall-layers in LES of atmospheric turbulence in wind farms, forests, and around mountains.

In order to illustrate a detailed picture of how vortex stretching may be active in subgrid turbulence, we consider LES of isotropic turbulence and compare the statistics of resolved eddies with respect to four subgrid models. We have investigated the invariants of the velocity-gradient, rate-of-strain, and rate-of-rotation. Velocity gradients are representative for small-scale intermittency, i.e. the mean separation of activated regions and intimately related to the direct interaction of large and small scales $[10,11]$. The concepts and ideas behind the Cauchy-Stokes decomposition of the velocity gradient tensor have been amply described in the published literature $[2,10,20]$. From the present mathematical analysis, one of the most interesting findings (from authors' point of view) is that the contribution to dissipation comes from the regions dominated by vortex sheets when the dissipation rate is obtained through vortex stretching rate. Using statistical properties of the subgrid-scale stress tensor $[13,20]$, a heuristic maybe that scale-locality of energy transfer holds if the energy flux depends on vortex stretching. We argue mathematically that the representation of vortex stretching may help subgrid models to account for local negative values in the energy flux. Such negative local energy flux is equivalent to representing the effects of energy 'backscatter'. In previous work, we considered similar idea of representing energy flux in LES of atmospheric boundary layer flows over arrays of buildings [18], mountains [17], and wind turbines [19].

Before moving further, it may be useful to introduce some illuminating ideas that the mean energy flux to small eddies is directly proportional to the mean rates of vortex stretching. Taylor [21] introduced the role of vortex stretching in driving energy from the largest to the smallest scales of turbulent motion [22]. For example, the lift - generated by a wing - and other fluid-solid interactions come from vortex motion. The fluid flow in the atmosphere and oceans can be described extremely well by collections of vortices. A mathematical expression that relates the energy flux to the stretching rate of large-scale vorticity was observed earlier, e.g., by Borue and Orszag [20]. Nevertheless, no prior work hypothesized to consider the rate of vortex stretching to compute the rate of energy dissipation in subgrid model for LES. Sagaut and Cambon [23] is a classical text, where one finds a rich mathematical details of vortex stretching, strain self-amplification, and energy dissipation. In [16], a statistical analysis of vortex stretching and strain self-amplification is considered in homogeneous isotropic turbulence. Past studies, such as [22,24,25], mostly focused on various important dynamical role of vortex stretching in turbulence (e.g. [1,2,2628]). Betchov [29] showed that the average strain skewness can also be related to average vortex stretching (e.g. see [10]). Shetty and Frankel [30] consider the 'spin-up' of vortex tube as a mechanism for forward energy cascade in the study of wall-bounded turbulence (see also [7]). To account for the effect of vortex-stretching in the subgrid model, Nicoud and Ducros [31] considered the rotation tensor along with the strain tensor in the wall-adapting local eddy viscosity (WALE) model for wall-bounded turbulence. Similarly, Refs $[17,18]$ considered the second invariant of the square of the velocity gradient tensor in the subgrid models for LES of atmospheric boundary layer flow over complex terrain [8]. In other words, the invariants of the velocity gradient tensor are very important particularly in turbulent flows, which help to understand necessary small scale information in closing the filtered Navier-Stokes system [2,31-34]. 
This article is organized as follows. In section 2, details of the LES method and subgrid models are discussed. The role of coherent vortices in the dissipation of subgrid-scale turbulence is discussed in section 3. Finally, section 4 provides some concluding remarks on future research directions.

\section{Methodology}

\subsection{Filtering approach and energy flux}

We consider an implicitly defined low-pass filter [35], where filtered velocity $\bar{u}_{i}$ is related to $u_{i}=\bar{u}_{i}+u_{i}^{\prime}$. Using the continuity equation

$$
\frac{\partial \bar{u}_{i}}{\partial x_{i}}=0
$$

to determine pressure $\bar{P}$, one obtains an approximation to $u_{i}(x, t)$ by solving the filtered Navier-Stokes equations,

$$
\frac{\partial \bar{u}_{i}}{\partial t}+\bar{u}_{j} \frac{\partial \bar{u}_{i}}{\partial x_{j}}=-\frac{1}{\rho} \frac{\partial \bar{P}}{\partial x_{i}}+\frac{\partial}{\partial x_{j}}\left(2 v \mathcal{S}_{i j}-\tau_{i j}\right)
$$

where $\rho$ and $v$ are the density and the kinematic viscosity, respectively. The filtering operation removes eddies smaller than a length scale $\Delta_{\text {les }}$. The residual (subfilter-scale) stress tensor $\tau_{i j}=\overline{u_{i} u_{i}}-\bar{u}_{i} \bar{u}_{j}$ accounts for the effects of such eddies. The residual stress may also be decomposed through the Germano identity [35], $\tau_{i j}=\mathcal{L}_{i j}+\mathcal{T}_{i j}$, where $\mathcal{L}_{i j}$ and $\mathcal{T}_{i j}$ are called Leonard and subgrid stresses, respectively. In classical Smagorinsky model, we define the eddy viscosity $v_{\tau}=\left(c_{s} \Delta\right)^{2}\left(\mathcal{S}_{i j} \mathcal{S}_{i j}\right)^{1 / 2}$ to approximate the stress [4]

$$
\tau_{i j}-\frac{1}{3} \tau_{k k} \delta_{i j}=-2 v_{\tau} \mathcal{S}_{i j}
$$

where $\mathcal{S}_{i j}=(1 / 2)\left(\mathcal{G}_{i j}+\mathcal{G}_{j i}\right)$ is the resolved rate of strain, $\delta_{i j}$ denotes the Kronecker delta, and the velocity gradient tensor $\mathcal{G}_{i j}=\partial_{j} u_{i}$ denotes partial derivatives of $u_{i}$ with respect to $x_{j}$. It is known that Smagorinsky's model, Eq (3), leads a good approximation to the subgrid stress; however, it is weakly correlated with the actual residual stresses. The energy flux $\Pi=-\mathcal{G}_{i j} \tau_{i j}$ of the Smagorinsky model takes the following form

$$
\Pi \approx c_{S}^{2} \Delta_{\text {les }}^{2} \mathcal{S}_{i j} \mathcal{S}_{j k} \mathcal{S}_{k i}
$$

which shows that the local behavior of the flux is governed by the dynamics of strain skewness, Trace $\left(\mathcal{S}^{3}\right)=\mathcal{S}_{i j} \mathcal{S}_{j k} \mathcal{S}_{k i}$. Generally speaking, the local dependence of the energy flux $\Pi$ on the strain and vorticity is not known a priori. Moreover, strain skewness and vortex stretching may be quite independent, and therefore a complete picture of the local energy transfer may require both [31].

The large-scale dynamics of energy balance in Eq (2) is given by (see [27])

$$
\frac{\partial E}{\partial t}+\frac{\partial \mathcal{J}_{j}}{\partial x_{j}}=-\Pi-2 \nu \mathcal{S}_{i j} \mathcal{S}_{i j}
$$

where the convective contribution to the energy flux of the large eddies is

$$
\mathcal{J}_{j}=E u_{j}+\bar{P} u_{j}+u_{i} \tau_{i j}-2 v \bar{u}_{i} \mathcal{S}_{i j}
$$

The rate of work done by the large-scale velocity gradient against the small-scale stress leads to the local energy flux $\Pi$ from large-scale eddies to small-scale ones. Negative local values of $\Pi$ cause 'backscatter' of energy from small to large eddies. Smagorinsky model, Eq (3), will always lead to a positive local energy flux without accounting for the effects of 'backscatter'. 


\subsection{Subgrid-scale turbulence}

The idea of dynamic Smagorinsky model is to account for local variation of $\Pi$, via $\mathrm{Eq}$ ( $3)$, through dynamic variation of $c_{s}(\boldsymbol{x}, t)$ in space and time. For completeness, we briefly present three approaches of finding dynamic variation of $c_{s}(x, t)$.

To implement the Langrangian-averaged dynamic model (hereinafter SGS-D) [5], we need to solve two transport equations, respectively, for $\mathcal{F}_{l m}$ and $\mathcal{F}_{m m}$, and finally,

$$
c_{s}^{2}(x, t)=\frac{\mathcal{F}_{l m}}{\mathcal{F}_{m m}} .
$$

The Lagrangian model solves following transport equations

$$
\frac{\partial \mathcal{F}_{l m}}{\partial t}+\overline{\boldsymbol{u}} \cdot \nabla \mathcal{F}_{l m}=\frac{1}{\mathcal{T}}\left(L_{i j} M_{i j}-\mathcal{F}_{l m}\right)
$$

and

$$
\frac{\partial \mathcal{F}_{m m}}{\partial t}+\bar{u} \cdot \nabla \mathcal{F}_{m m}=\frac{1}{\mathcal{T}}\left(M_{i j} M_{i j}-\mathcal{F}_{l m}\right),
$$

where the parameter $\mathcal{T}=\Delta\left(\mathcal{F}_{l m} \mathcal{F}_{m m}\right)^{-1 / 8}$ controls the memory length of the Lagrangian averaging (see [5] for other options of $\mathcal{T}$ ). In the above equations, the Leonard stress $L_{i j}$ is directly computed from the implicitly filtered velocity $\bar{u}_{i}$ through a second filtering operation considered between $\Delta$ and $n \Delta$ (usually $n=2$ ), and $M_{i j}$ accounts for the error associated with the use of Smagorinsky model in Germano identity, where

$$
L_{i j}={\widehat{\overline{u_{i}}}}_{i}-\widehat{\bar{u}}_{i} \widehat{\bar{u}}_{j} \quad \text { and } \quad M_{i j}=2 \Delta^{2}\left[\widehat{|\mathcal{S}| \mathcal{S}_{i j}}-4 \beta|\widehat{\mathcal{S}}| \widehat{\mathcal{S}}_{i j}\right] \text {. }
$$

Here, $\beta$ is a parameter to control scale dependency in the presence of complex solid obstacles. For the results presented in this article, $\beta=1$.

An approach of incorporating variations in the eddy viscosity (hereinafter SGS-C), commonly used in atmospheric boundary layer simulations [36-39], is to consider local variations of subgrid TKE, $k_{\mathrm{sgs}}=(1 / 2) L_{k k}$. This model solves the following transport equation,

$$
\frac{\partial k_{\mathrm{sgs}}}{\partial t}+\bar{u}_{j} \frac{\partial k_{\mathrm{sgs}}}{\partial x_{j}}=-\tau_{i j} \mathcal{S}_{i j}-\varepsilon+\frac{\partial}{\partial x_{j}}\left[\left(v_{\tau}+v\right) \frac{\partial k_{\mathrm{sgs}}}{\partial x_{j}}\right] .
$$

The eddy viscosity and dissipation are written in terms of $k_{\mathrm{sgs}}$ and $\Delta_{\text {les }}$ (see $\left.[17,39]\right)$ :

$$
v_{\tau}=C_{k} \sqrt{k_{\mathrm{sgs}}} \Delta_{\mathrm{les}}, \quad \varepsilon=C_{\epsilon} k_{\mathrm{sgs}}^{3 / 2} / \Delta .
$$

Fixed values of $c_{k} \sim 0.1$ and $c_{\epsilon} \sim 0.19+0.74 \ell / \Delta_{\text {les }}$ are commonly used in meteorological applications [39]. Clearly, the resulting eddy viscosity will fluctuate dynamically, which is due to the subgrid scale dynamics introduced by the above transport equation, Eq (5). The parameter $\ell$ aims to model local variation of length scales in the presence of stratification (otherwise $\ell=\Delta_{\mathrm{les}}$ ).

There have been several other variants of the above $k_{\mathrm{sg}}$-based model. One of which (hereinafter SGS-B) employs the Germano identity in order to dynamically estimate both $C_{k}$ and $C_{\epsilon}$ (e.g. see $[17,40]$ for details). The accuracy of three dynamic subgrid models discussed above was thoroughly scrutinized in the literature, particularly in the context of wall-modeled LES e.g. see $[3,9,18,41]$.

\subsection{Vortex stretching and subgrid-scale stress}

Consider the Taylor series expansion of $\bar{u}_{i}(\boldsymbol{x}, t)$ about a local average $\tilde{\tilde{u}}_{i}(\boldsymbol{r}, t)$, where

$$
\bar{u}_{i}(\boldsymbol{x}, t) \approx \tilde{\bar{u}}_{i}(\boldsymbol{r}, t)+\left[\frac{\partial \bar{u}_{i}}{\partial x_{j}}(\boldsymbol{r}, t)\right](\boldsymbol{x}-\boldsymbol{r}) .
$$


Considering the local average $\tilde{\tilde{u}}_{i}(r, t)$ with respect to a box of size $2 \Delta_{\text {les, }}$, the Leonard component of the residual stress [42] is

$$
\tau_{i j}^{L}=c_{k} \Delta_{\text {les }}^{2} \mathcal{G}_{i k} \mathcal{G}_{j k} .
$$

Note that we have used the symbol $\tau_{i j}^{L}$ instead of $\mathcal{L}_{i j}$. The most important characteristics of $\tau_{i j}^{L}$ is its connection to vortex stretching - a mechanism that plays an important role in turbulence energy cascade. Following Betchov's theory [29], and considering that Trace $\mathcal{G}_{i j}=\mathcal{G}_{i i}=0$, the energy flux $\Pi=\mathcal{G}_{i j} \tau_{i j}^{L}$ is expressed as

$$
\Pi=c_{k} \Delta^{2}\left[- \text { Trace } \mathcal{S}^{3}+\frac{1}{4} \omega_{i} \omega_{j} \mathcal{S}_{i j}\right]
$$

where $\omega_{i}=\epsilon_{i j k} \mathcal{G}_{j k}$ denotes the vorticity vector. In fact, appealing to the pioneering Betchov theory [29], it follows that the local energy flux is positive if the skewness of the filtered strain matrix is negative and the vortex stretching term $\omega_{i} \omega_{j} \mathcal{S}_{i j}$ is positive. Using Betchov's relation (see [20,29]), such as $-\left\langle\right.$ Trace $\left.\mathcal{S}^{3}\right\rangle=(3 / 4)\left\langle\omega_{i} \omega_{j} \mathcal{S}_{i j}\right\rangle$, the dissipation of TKE in the Smagorinsky model leads to $\langle\Pi\rangle=c_{k} \Delta^{2}\left\langle\omega_{i} \omega_{j} \mathcal{S}_{i j}\right\rangle$. It is worth mentioning that vortex tubes tend to induce a tensile stress in the plane orthogonal to their axis [2]. In other words, stretching of vortex tubes requires large-scale strain to loose energy by overcoming this opposing stress [16].

For brevity, we ignore the factor $c_{k} \Delta_{\text {les }}^{2}$ in Eq (6). Discarding the effects of the rotation tensor in $\mathcal{G}_{i k} \mathcal{G}_{j k}=\mathcal{G}_{i k} \mathcal{G}_{k j}+\mathcal{G}_{i k} \mathcal{R}_{j k}$ the second invariant of the deviatoric Leonard stress tensor

takes the form

$$
\tau_{i j}^{L^{d e v}}=\frac{1}{2}\left[\tau_{i j}^{L}+\tau_{j i}^{L}\right]-(1 / 3) \tau_{k k}^{L} \delta_{i j}
$$

$$
-\frac{1}{2} \tau_{i j}^{L^{d e v}} \tau_{i j}^{L^{d e v}}=-\frac{1}{4}\left[\mathcal{S}_{i j} \omega_{j} \mathcal{S}_{i k} \omega_{k}+\frac{1}{3}\left(\mathcal{G}_{i j} \mathcal{G}_{i j}\right)^{2}\right] .
$$

Now, consider dimensional reasoning similar to [31], which leads to the following form of the subgrid-scale TKE (hereinafter SGS-A),

$$
k_{\mathrm{sgs}}=\frac{\Delta_{\text {les }}^{2}\left(\frac{1}{2} \mathcal{S}_{i j} \omega_{j} \mathcal{S}_{i k} \omega_{k}+\frac{2}{3}\left(\mathcal{G}_{i j} \mathcal{G}_{i j}\right)^{2}\right)^{3}}{\left[\left(\mathcal{S}_{i j} \mathcal{S}_{i j}\right)^{5 / 2}+\left(\frac{1}{2} \mathcal{S}_{i j} \omega_{j} \mathcal{S}_{i k} \omega_{k}+\frac{2}{3}\left(\mathcal{G}_{i j} \mathcal{G}_{i j}\right)^{2}\right)^{(5 / 4)}\right]^{2}} .
$$

Consider Eq (8) to compute $k_{\mathrm{sgs}}$ without solving Eq (5). Thus, the subgrid-scale stress tensor may be expressed as

$$
\tau_{i j}-\frac{1}{3} \tau_{k k} \delta_{i j}=c_{k} \Delta_{\mathrm{les}} \sqrt{k_{\mathrm{sgs}}} \mathcal{S}_{i j} .
$$

Considering Eqs (8) and (9), we see that local values of $\tau_{i j}$ is dynamically adjusted according to the strength of vortex stretching and the relative dominance of strain $\mathcal{S}_{i j}$ over rotation $\mathcal{R}_{i j}$. We can assign a value of the parameter $C_{k}$ according to a desired global rate of dissipation. An advantage is that the eddy viscosity, Eq (9), learns the subgrid-scale energy dissipation rate from the statistics of velocity gradient tensor. For example, an idealized vortex tube will exert a tensile stress on the surrounding straining motion when it is stretched [2]. Consideration of Eq (9) as a subgrid model is equivalent to transferring energy via work done against vortex stretching.

There are several mathematical illustration of how the straining motion can intensify idealized vortex tubes and sheets. DNS and LES of actual turbulent flow resemble such idealized vortex tubes of diameters ranging from Taylor micro-scale $(\lambda)$ down to Kolmogorov micro-scale $(\eta)$. Thus, we can assess the main advantages of the vortex stretching-based model, Eq (9) via a statistical quantification of the comparision among four subgrd models using the decay of homogeneous turbulence. 


\section{Result}

Consider a fully developed synthetic turbulent flow field $u_{i}(\boldsymbol{x}, 0), i=1,2,3$, which follows the von Karman spectral model

$$
E(k)=\alpha\left(\frac{k}{k_{0}}\right)^{4}\left(1+\frac{k^{2}}{k_{0}^{2}}\right)^{-17 / 6} e^{-2 k^{2} / k_{\eta}^{2}}
$$

where $k_{\eta}=\left(\langle\epsilon\rangle / v^{3}\right)^{1 / 4}$. It satisfies the energy distribution of $k^{4}$ for the large eddies which contain most of the energy and reproduces the $k^{-5 / 3}$ power-law in the inertial subrange. We have chosen $\alpha$ to get a desired value of $\left|\boldsymbol{u}_{0}\right|=\int_{\Omega}|\boldsymbol{u}(\boldsymbol{x}, t)| d \boldsymbol{x}$, i.e., $E(0)=(1 / 2)\left|\boldsymbol{u}_{0}\right|^{2}$. Table 1 compares the average values of Taylor micro scale $\lambda=\sqrt{15 v u_{r m s}^{2} /\langle\epsilon\rangle}$, Kolmogorov's micro scale $\eta=\left(v^{3} /\langle\epsilon\rangle\right)^{1 / 4}$, Taylor Reynolds number $\mathcal{R} e_{\lambda}=u_{r m s} \lambda / v$, and the viscous dissipation rate $\langle\epsilon\rangle$. The integral scale Reynolds number $\mathcal{R} e=\left|\boldsymbol{u}_{0}\right| 2 \pi / \nu$ may also be related to the Taylor scale Reynolds number as $\mathcal{R}_{\lambda} \sim \sqrt{10 \mathcal{R} e / 3}$. For $\left|\boldsymbol{u}_{0}\right|=10[\mathrm{~m} / \mathrm{s}]$, we have $\mathcal{R} e=3.14 \times 10^{6}$ and $\mathcal{R}_{\lambda} \sim 3.25 \times 10^{3}$. Here, $\langle\cdot\rangle$ denotes a volume average with respect to the entire computational domain. The number of cells $128^{3}$ in the domain $[0,2 \pi]^{3}$ implies that the cutoff wavenumber is 32 for a filter width twice as large as the grid spacing.

\begin{tabular}{llcccc}
\hline \hline Model & Remark & $\lambda$ & $\eta$ & $\mathcal{R} e_{\lambda}$ & $\langle\epsilon\rangle$ \\
\hline SGS-A & Nicoud and Ducros [31] & 0.1105 & $5.9719 \mathrm{e}-04$ & 8837 & 0.0629 \\
SGS-B & Yoshizawa [40] & 0.0796 & $5.0677 \mathrm{e}-04$ & 6365 & 0.1231 \\
SGS-C & Deardorff [37] & 0.1068 & $5.8718 \mathrm{e}-04$ & 8546 & 0.0673 \\
SGS-D & Meneveau et al. [5] & 0.0880 & $5.3308 \mathrm{e}-04$ & 7043 & 0.0991 \\
\hline \hline
\end{tabular}

Table 1: A comparison for the values of $\lambda, \eta, \mathcal{R} e_{\lambda}$, and $\langle\epsilon\rangle$ predicted from the LES data corresponding to 4 subgrid models.

\subsection{Skewness and velocity gradient tensor}

In LES of complex engineering and geophysical flows, the interplay of the strain and vorticity fields is of great importance in the transfer of energy from large to small scales $[2,16]$. The vorticity vector $\boldsymbol{\omega}$ shows a preferred alignment with the intermediate eigenvector of the strain rate tensor. For this reason, vortex stretching vector and velocity gradient skewness are among the central quantities thought responsible for the mean rate of enstrophy production.

The second and the third invariants, respectively, $Q^{\mathcal{G}}=-(1 / 2) \mathcal{G}_{i j} \mathcal{G}_{i j}$ and $R^{\mathcal{G}}=$ $(1 / 3) \mathcal{G}_{i j} \mathcal{G}_{j k} \mathcal{G}_{k i}$, of the velocity gradient tensor $\mathcal{G}_{i j}$ in incompressible flow take the form:

$$
Q^{\mathcal{G}}=-\frac{1}{2} \mathcal{S}_{i j} \mathcal{S}_{i j}+\frac{1}{4} \omega_{i} \omega_{i}, \quad R^{\mathcal{G}}=-\frac{1}{3}\left(\mathcal{S}_{i j} \mathcal{S}_{i k} \mathcal{S}_{k i}+\frac{3}{4} \omega_{i} \omega_{j} \mathcal{S}_{i j}\right) .
$$

The second invariant $Q^{\mathcal{G}}$ accounts for the relative importance of dissipation rate over enstrophy. The third invariant $R^{\mathcal{G}}$ accounts for an overall effects of strain skewness and enstrophy production rate. These two invariants can be expressed in the form of divergence:

$$
Q^{\mathcal{G}}=-\frac{1}{2} \frac{\partial}{\partial x_{j}}\left[u_{i} \frac{\partial u_{j}}{\partial x_{j}}\right], \quad R^{\mathcal{G}}=\frac{1}{3} \frac{\partial}{\partial x_{i}}\left[\frac{\partial u_{i}}{\partial x_{j}} \frac{\partial u_{j}}{\partial x_{k}} u_{k}-\frac{1}{2} u_{i} \frac{\partial u_{k}}{\partial x_{j}} \frac{\partial u_{j}}{\partial x_{k}}\right]
$$

Based on these quantities, the volume average of each of the invariants vanishes if the quantities inside the square bracket [·] vanish on the boundary or a periodic boundary condition is assumed. Now, consider the Betchov relation, $\left\langle Q^{\mathcal{G}}\right\rangle=0$, which states that the production of enstrophy will enhance the overall dissipation rate [43]. The mean enstrophy 
$\left\langle\omega_{i} \omega_{i}\right\rangle$ and the mean rate of enstrophy production $\left\langle\omega_{i} \omega_{j} \mathcal{S}_{i j}\right\rangle$ can be written in terms of the principal strain rates:

$$
\left\langle\omega_{i} \omega_{i}\right\rangle=2\left\langle\lambda_{1}^{2}+\lambda_{2}^{2}+\lambda_{3}^{2}\right\rangle, \quad\left\langle\omega_{i} \omega_{j} \mathcal{S}_{i j}=-\frac{4}{3}\left\langle\lambda_{1}^{3}+\lambda_{2}^{3}+\lambda_{3}^{3}\right\rangle,\right.
$$

where $\lambda_{i}$ 's are eigenvalues of the strain tensor. Following Betchov [29], we get for isotropic turbulence

$$
\left\langle\left(\frac{\partial u_{1}}{\partial x_{1}}\right)^{2}\right\rangle=\frac{2}{15}\left\langle\lambda_{1}^{2}+\lambda_{2}^{2}+\lambda_{3}^{2}\right\rangle, \quad\left\langle\left(\frac{\partial u_{1}}{\partial x_{1}}\right)^{3}\right\rangle=\frac{8}{105}\left\langle\lambda_{1}^{3}+\lambda_{2}^{3}+\lambda_{3}^{3}\right\rangle .
$$

The above quantities provide a link between the mean rate of enstrophy production by vortex stretching, $\left\langle\omega_{i} \omega_{j} \mathcal{S}_{i j}\right\rangle$ and the skewness factor of the velocity gradient, i.e. $S_{0}=$ $\left\langle\left(\frac{\partial u_{1}}{\partial x_{1}}\right)^{3}\right\rangle /\left\langle\left(\frac{\partial u_{1}}{\partial x_{1}}\right)^{2}\right\rangle^{3 / 2}$ such that

$$
S_{0}=-\frac{6 \sqrt{15}\left\langle\omega_{i} \omega_{j} \mathcal{S}_{i j}\right\rangle}{7\left\langle\omega_{i} \omega_{i}\right\rangle^{3 / 2}}
$$

A negative value of skewness tells us that the net effect of the strain field is to create enstrophy. Said in other words, average enstrophy production by vortex stretching, $\left\langle\omega_{i} \omega_{j} \mathcal{S}_{i j}\right\rangle$, is positive.

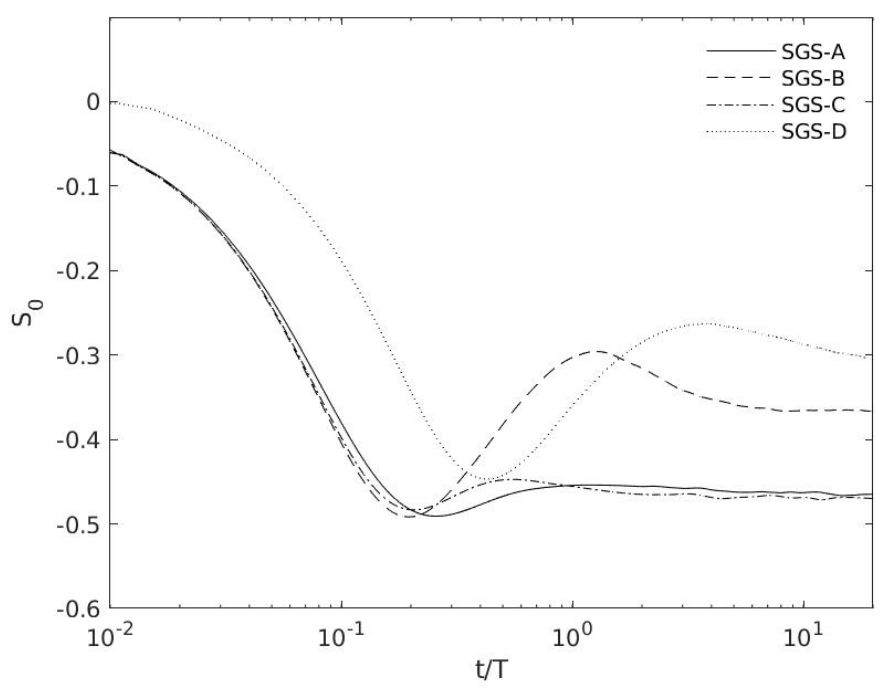

Figure 1. A comparison for the time history of the velocity gradient skewness $S_{0}$ with respect to four subgrid models.

Figure 1 compares the variation of $S_{0}$ as a function of the dimensionless time $t / T$, where $T=\left|\boldsymbol{u}_{0}\right| /$ L. The LES data for two of the models, SGS-A and SGS-C, indicate that the skewness evolves to an equilibrium value of nearly -0.4 for $t / T>0.1$. A value of $S_{0} \approx-0.4 \pm 0.1$ is usually observed in laboratory measurements of isotropic turbulence. The result tells us two important messages. First, an approximate balance between the production of enstrophy and viscous dissipation is characterized by the velocity gradient tensor (for a further discussion, see [31]). Second, the net effect of vortex stretching is to transfer the kinetic energy that is associated with the production of enstrophy, indicating a natural tendency that creates smaller scales. In other words, the existence of vortices on all possible scales, e.g. [44], indicates that the enstrophy production by vortex stretching corresponds to the energy transfer from large to small scales [2]. 


\subsection{Second moment of the velocity field}

It is widely accepted that the temporal evolution of the resolved turbulence kinetic energy, $E(t)=(1 / 2)\langle\boldsymbol{u} \cdot \boldsymbol{u}\rangle$ follows Kolmogorov's decay law $E(t) \sim\left(t-t_{0}\right)^{-10 / 7}[2,4]$.
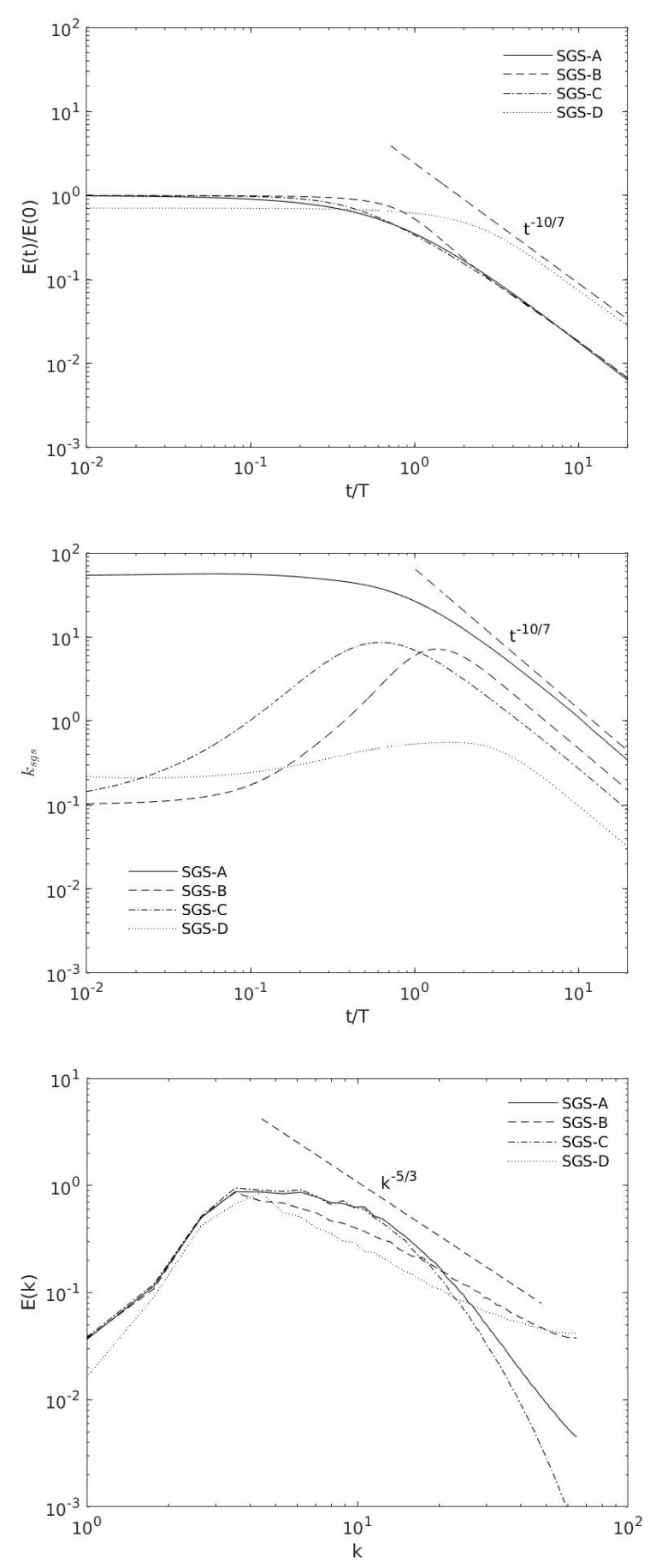

Figure 2. A comparison of the second moment of the velocity field with respect to 4 subgrid models, Kolmogorov's decay law $t^{-10 / 7}$, and power law, $k^{-5 / 3}$. (a) $E(t) / E(0)$ and $t^{-10 / 7}$. (b) $k_{\mathrm{sgs}}$ and $t^{-10 / 7}$. (c) $E(k)$ at $t / T=3$ and $k^{-5 / 3}$.

Figure 2a compares the decay of the resolved energy among four models. The resolved energy $E(t)$ is the sum of filtered energy and subgrid-scale TKE, where the subgrid-scale TKE, $k_{\text {sgs }}=(1 / 2) \operatorname{Trace}\left({\overline{u_{i}}}_{\mathrm{u}_{j}}-\overline{\mathrm{u}}_{\mathrm{i}} \overline{\mathrm{u}}_{\mathrm{j}}\right)$ is the contribution from subgrid models. Figure $2 \mathrm{~b}$ compares $k_{\mathrm{sgs}}$ among four subgrid models. It is interesting to observe that the vortex 
stretching-based model (SGS-A) has captured a relatively large amount of TKE, $k_{\mathrm{Sgs}}$. Figure 2(c) compares the energy spectrum $E(k)$ with respect to four subgrid models. The distribution of energy in Fourier space, $E(k)$, follows Kolmogorov's power law $k^{-5 / 3}$.

\subsubsection{Viscous dissipation}

Using Betchov's result [2,29] on the second invariant of the velocity gradient tensor, i.e. $\left\langle Q^{\mathcal{G}}\right\rangle=0$, the viscous dissipation rate $\epsilon=2 v \mathcal{S}_{i j} \mathcal{S}_{i j}$ is correlated with mean enstrophy such that $\langle\epsilon\rangle \equiv 2 v\left\langle\mathcal{S}_{i j} \mathcal{S}_{i j}\right\rangle=v\left\langle\omega_{i} \omega_{i}\right\rangle$. We have calculated the resolved energy flux $\Pi_{\mathrm{FD}}$ by applying finite difference method on the time series of resolved energy $E(t)$. The energy flux modeled in LES is $\Pi \approx-\tau_{i j} \mathcal{S}_{i j}$. The correlation between $\left\langle\Pi_{\mathrm{FD}}\right\rangle=d E / d t$ and $\langle\Pi\rangle=\left\langle\tau_{i j} \mathcal{S}_{i j}\right\rangle$, as well as that between viscous dissipation and $v\left\langle\omega^{2}\right\rangle$ are shown in Fig 3.

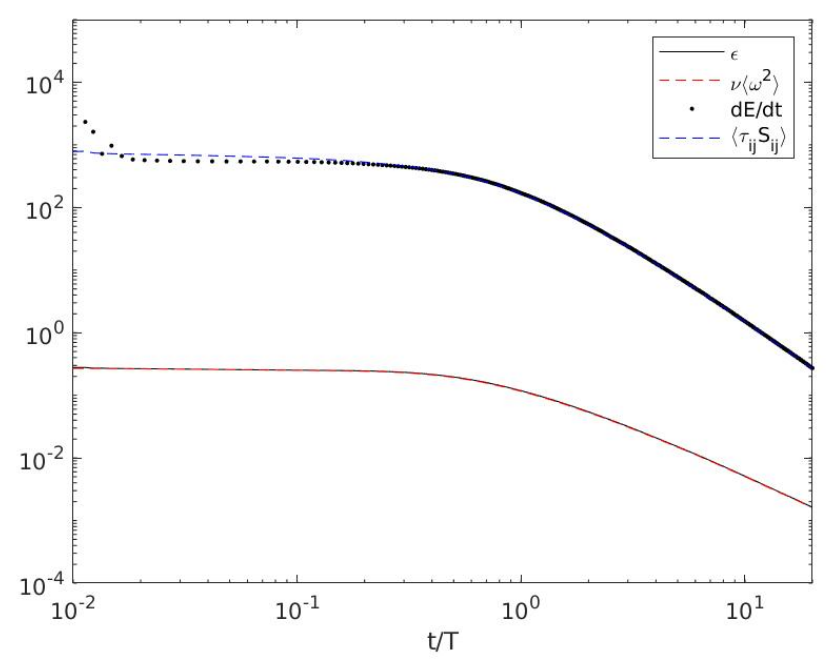

Figure 3. A plot of the time series of the rate of change of the resolved energy, $d E / d t$ (block ...), the energy flux $\left\langle\tau_{i j} \mathcal{S}_{i j}\right\rangle$ (black - - -), the viscous dissipation rate $\epsilon$ (red - - -), and the mean enstrophy $\left\langle\omega^{2}\right\rangle$ (black - ).

In LES, modeled rate of energy transfer from large to small scales, $-\tau_{i j} \mathcal{S}_{i j}$, is always positive for eddy viscosity models considered in this study. In other words, the rate of loss of resolved turbulence kinetic energy, $d E / d t$, is expected to be correlated with the transfer of energy in decaying turbulence. Figure 3 compares the rate of dissipation and the transfer of energy by turbulence. Such a correlation has been observed in the velocity field collected from four cases of LES considered in this article. A close agreement between $d E / d t$ and $\left\langle\tau_{i j} \mathcal{S}_{i j}\right\rangle$ in Figure 3 indicates that vortex stretching does not oppose the energy dissipation for the present test case. 
3.3. Statistics, vortices, stretches, and whirls of turbulence

3.3.1. Dynamics of filtered velocity gradients

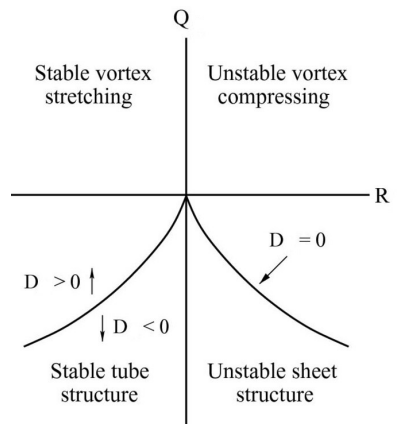

(a)

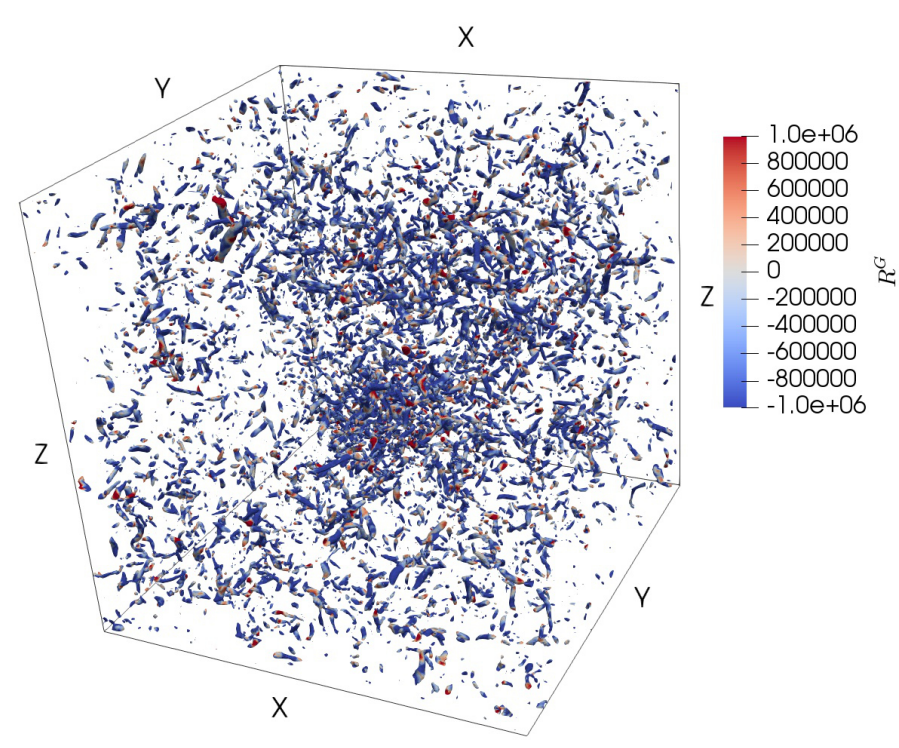

(b)

Figure 4. (a) Schematic illustration of three-dimensional incompressible flow based on the invariant map $\left(R^{\mathcal{G}}, Q^{\mathcal{G}}\right)$, which states that vorticity dominates over rate of strain where the region $\left((27 / 4)\left(R^{\mathcal{G}}\right)^{2}+\left(Q^{\mathcal{G}}\right)^{3}>0\right)$ [33]. (b) Isosurface plot of $20 \%$ positive deviation of $Q^{\mathcal{G}}$, which is colored by $R^{\mathcal{G}}$ for a turbulent flow simulated at $256^{3}$ grid points using the vortex stretching-based model SGS-A, Eq (9).

The $\left(R^{\mathcal{G}}, Q^{\mathcal{G}}\right)$ diagram in Fig $4 a$ indicates that a fluid region with $Q^{\mathcal{G}}>0$ consists of isolated whirled eddies intermittently dispersed with coherent activity of high vorticity. These eddies are stretched (compressed) if $R^{\mathcal{G}}<0\left(R^{\mathcal{G}}>0\right)$. Kelvin-Helmholtz theorem implies that the vorticity $\omega(t)$ of a vortex tube of length $L(t)$ increases as it stretches; i.e. conservation of circulation is equivalent to i.e $\omega(t) / \omega(0)=L(t) / L(0)$. Fig $4 b$ displays approximately $20 \%$ positive deviation of $Q^{\mathcal{G}}$ colored with $R^{\mathcal{G}}$ where the turbulence field has been obtained by LES at a resolution of $256^{3}$ grid points and Reynolds number $\mathcal{R} e=3.14 \times$ $10^{6}$. It indicates that large-magnitude vorticity mostly occurs in tubes $\left(R^{\mathcal{G}}<0\right)$, which are intermittently dispersed with coherent regions of high vorticity $\left(\omega(t)=\sqrt{4 Q^{\mathcal{G}}+2 \operatorname{Tr} \mathcal{S}^{2}}\right)$. Vortex tubes are surrounded by high strain rate (in empty region $Q^{\mathcal{G}}<0$ ). It is well known that vortex tubes appear like 'spaghetti on a plate' - a details of which are not resolved in LES because the number of grid points $256^{3}$ considered in the simulation is relatively small with respect to $\mathcal{R} e=3.14 \times 10^{6}$.

The velocity gradient tensor illuminates the local topology of the flow resolved at the length scale $\Delta_{\text {les }}$. The equation of the velocity gradient tensor is derived by taking the gradient of Eq (2),

$$
\frac{D \mathcal{G}_{i j}}{D t}=-\left(\mathcal{G}_{i k} \mathcal{G}_{k j}-\frac{1}{3} \mathcal{G}_{m n} \mathcal{G}_{n m} \delta_{i j}\right)-\left(\frac{\partial^{2} \bar{P}}{\partial x_{i} \partial x_{j}}-\frac{1}{3} \nabla^{2} \bar{P} \delta_{i j}\right)+v \frac{\partial^{2} \mathcal{G}_{i j}}{\partial x_{j} \partial x_{j}}-\frac{\partial^{2} \tau_{i j}}{\partial x_{j} \partial x_{j}}
$$

where we have assumed that $\nabla^{2} \bar{P}=2 Q^{\mathcal{G}}$. Terms on the right hand side of Eq (10), except the two within the first pair of round brackets, represent the interaction with the velocity gradients of other surrounding fluid particles. The most important feature of the dynamics of the velocity gradient tensor is given by

$$
\frac{D}{D t}\left(\frac{1}{2} \mathcal{G}_{i j} \mathcal{G}_{i j}\right)=\frac{1}{4} \omega_{i} \mathcal{S}_{i j} \omega_{j}-\mathcal{S}_{i j} \mathcal{S}_{j k} \mathcal{S}_{k i}
$$


Clearly, the local rate of production of the velocity gradient depends on the alignment between the vorticity and the eigenvector of strain rate tensor, $\omega_{i} \mathcal{S}_{i j} \omega_{j}=\omega^{2} \lambda_{i}\left(\boldsymbol{e}_{i} \cdot \boldsymbol{e}_{\omega}\right)^{2}$. Here, $e_{i}$ and $e_{\omega}$ are strain rate eigenvectors and vorticity unit vectors, respectively. Based on Betchov's relations [29], the average amount of strain self-amplification is three times the vortex stretching; i.e.. $(3 / 4)\left\langle\omega_{i} \mathcal{S}_{i j} \omega_{j}\right\rangle=-\left\langle\mathcal{S}_{i j} \mathcal{S}_{j k} \mathcal{S}_{k i}\right\rangle$. Hence

$$
\frac{D}{D t}\left\langle\mathcal{G}_{i j} \mathcal{G}_{i j}\right\rangle=2\left\langle\omega_{i} \mathcal{S}_{i j} \omega_{j}\right\rangle
$$

Thus, Eq (10) indicates that the energy cascade is driven by vortex stretching, which also depends on strain self-amplification (see also [16]).

\subsubsection{Statistics}

A compact and relatively convenient way to analyze the influence of the filtered velocity gradient tensor is the following five-dimensional dynamical systems of the invariants [8]:

$$
\begin{aligned}
\frac{d Q^{\mathcal{G}}}{d t} & =-3 R^{\mathcal{G}} \\
\frac{d R^{\mathcal{G}}}{d t} & =\frac{2}{3}\left(Q^{\mathcal{G}}\right)^{2} \\
\frac{d Q^{S}}{d t} & =-2 R^{S}-R^{\mathcal{G}} \\
\frac{d R^{S}}{d t} & =\frac{2}{3} Q^{\mathcal{G}} Q^{S}+\frac{1}{4} \mathcal{V}^{2} \\
\frac{d \mathcal{V}^{2}}{d t} & =-\frac{16}{3}\left(R^{S}-R^{\mathcal{G}}\right) Q^{\mathcal{G}} .
\end{aligned}
$$

Here, $Q^{S}=-(1 / 2) \mathcal{S}_{i j} \mathcal{S}_{i j}, R^{S}=-(1 / 3) \mathcal{S}_{i j} \mathcal{S}_{j k} \mathcal{S}_{k i}$, and the magnitude of the vortex stretching vector is $\mathcal{V}=|\mathcal{S} \boldsymbol{\omega}|$. It can also be seen that the first two of the system of equations are decoupled from the remaining three equations. Clearly, $Q^{\mathcal{G}}$ increases if $R^{\mathcal{G}}<0$, and vice versa. Notably, it is a high-dimensional dynamical system with $5 \times 256^{3}$ degrees of freedom.

The histograms of $R^{\mathcal{G}}$ and $Q^{\mathcal{G}}$ are shown in Figs 5-6. For each of the four subgrid models tested, the histograms are distributed with high values of Kurtosis. In the statistical theory, the Kurtosis of a probability distribution measure the level of intermittency of an event. Observed Kurtosis factors of $R^{\mathcal{G}}$ and $Q^{\mathcal{G}}$ are larger than 3, which implies a non-Gaussian leptokurtic distribution of the event regardless of the choice of the subgrid model. From the histograms of $\mathcal{R}^{\mathcal{G}}$, the relatively large Kurtosis $(56-60)$ implies that the energy flux in two of the models, i.e. SGS-A and SGS-B, is relatively more intermittent. On the other hand, the histograms of $\mathcal{R}^{\mathcal{G}}$ form the other two models, i.e. SGS-C and SGS-D, indicates a relatively Gaussian, but leptokurtic distribution of the energy flux. 


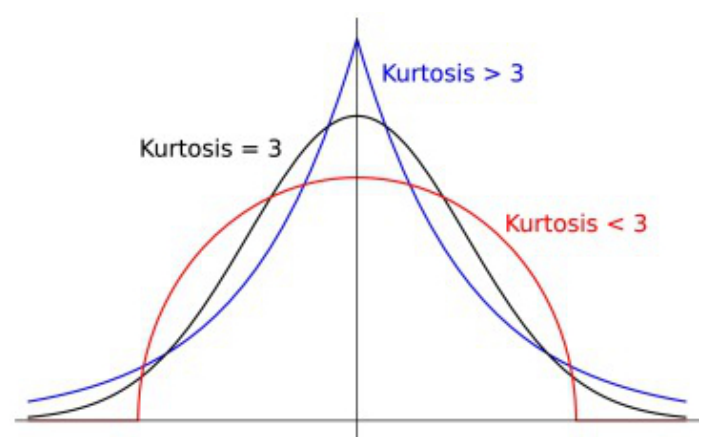

(a)

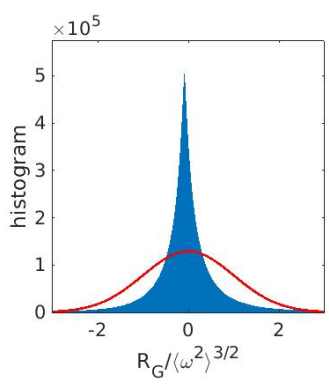

(b) Kurtosis $=60$

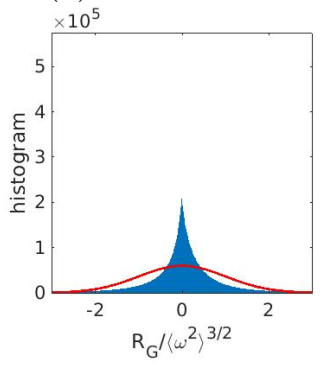

(b) Kurtosis $=20$

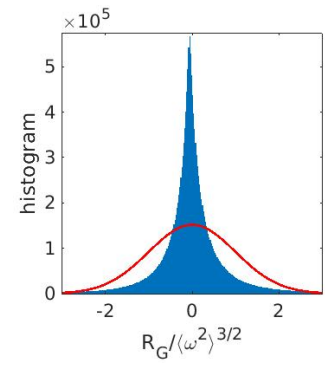

(c) Kurtosis $=56$

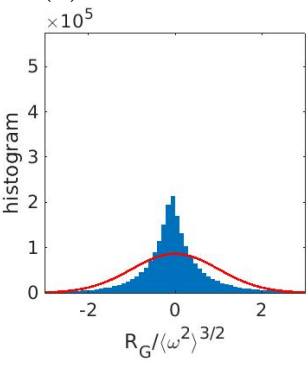

(c) Kurtosis $=23$

Figure 5. (a) A schematic illustration of mesokurtic (Kurtosis = 3), leptokurtic (Kurtosis > 3) and platykurtic (Kurtosis $<3$ ) distribution. $(b-e)$ Plots of the probability density function of $R_{G}$, the third invariant of the velocity gradient tensor $\mathcal{G}$ with respect to subgrid models SGS-A (b), SGS-B $(c)$, SGS-C $(d)$, and SGS-D $(e)$ (see Table 1 ). 


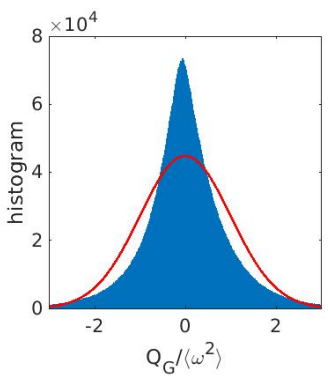

(b) Kurtosis $=8$

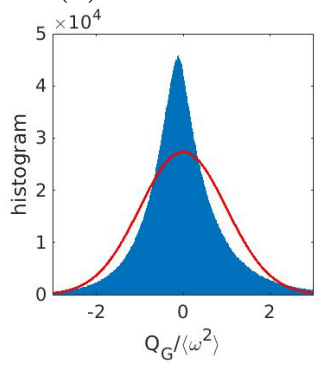

(b) Kurtosis $=8$

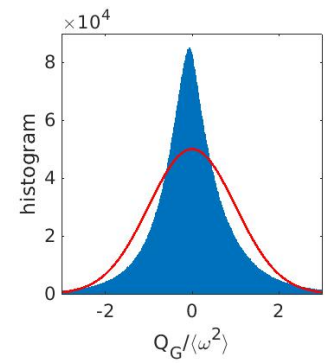

(c) Kurtosis $=8$

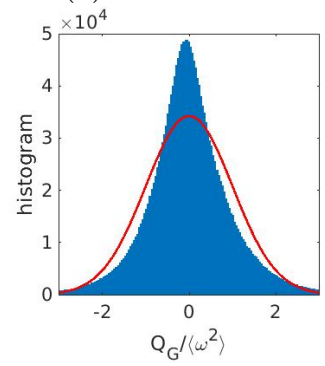

(c) Kurtosis $=6$

Figure 6. Plots of the probability density function of $Q_{G}$, the second invariant of the velocity gradient tensor $\mathcal{G}$ with respect to subgrid models SGS-A $(a)$, SGS-B $(b)$, SGS-C $(c)$, and SGS-D $(d)$ (see Table 1).

\subsubsection{Energy flux, vortex stretching, and strain skewness}

In Fig 7, the joint PDF of $R^{\mathcal{G}}$ and $Q^{\mathcal{G}}$ shows that the bulk of the instantaneous velocity field is primarily in two quadrants: $R^{\mathcal{G}}<0, Q^{\mathcal{G}}>0$ and $R^{\mathcal{G}}>0, Q^{\mathcal{G}}<0$. In the literature, the $\left(R^{\mathcal{G}}, Q^{\mathcal{G}}\right)$ map was thoroughly scrutinized by many researchers suggesting that there is a sharp division between the vortex dominated region $Q^{\mathcal{G}}>-3 \sqrt[3]{\left(R^{\mathcal{G}}\right)^{2} / 4}$ and strain dominated region $Q^{\mathcal{G}}<-3 \sqrt[3]{\left(R^{\mathcal{G}}\right)^{2} / 4}$. The $\left(R^{\mathcal{G}}, Q^{\mathcal{G}}\right)$ map shows a strong negative correlation, i.e. $Q^{\mathcal{G}} R^{\mathcal{G}}<0$. The $\left(R^{\mathcal{G}}, Q^{\mathcal{G}}\right)$ map provides some quantitative measure of the energy flux from large- to small-scales, Eq (7), i.e. $\Pi=c_{k} \Delta^{2}\left[\mathcal{R}^{\mathcal{G}}+(1 / 3) \omega_{i} \omega_{j} \mathcal{S}_{i j}\right]$ and $\langle\Pi\rangle=c_{k} \Delta^{2}\left\langle\omega_{i} \omega_{j} \mathcal{S}_{i j}\right\rangle$. The the $\left(R^{\mathcal{G}}, Q^{\mathcal{G}}\right)$ map among four subgrid models support a good correlation between the energy casecade and vortex stretching, and that two most common topological states of the flow are vortex stretching $\omega_{i} \omega_{j} \mathcal{S}_{i j}>0$ and unstable sheet $\lambda_{1} \lambda_{2} \lambda_{3}<0[8,33]$. 


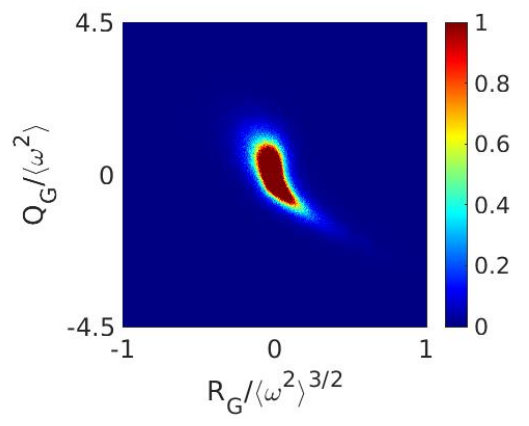

(a) SGS-A

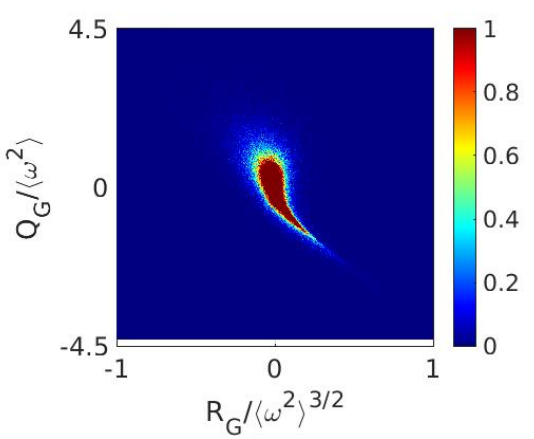

(c) SGS-C

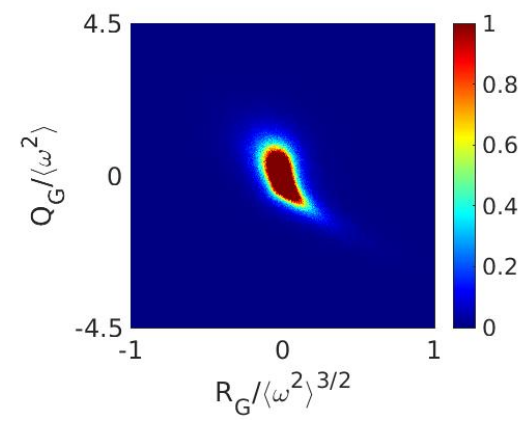

(b) SGS-B

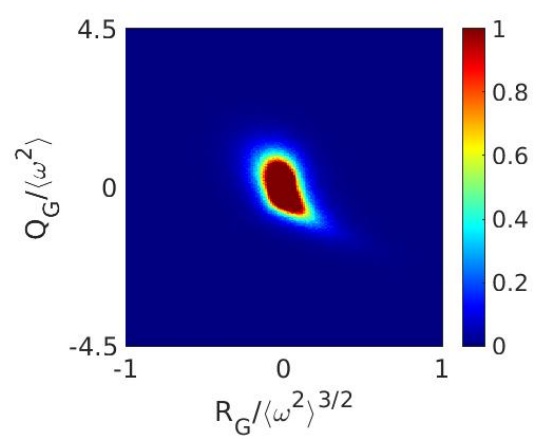

(d) SGS-D

Figure 7. Plots of the joint probability density function of two invariants $Q_{G}$ and $R_{G}$ of the velocity gradient tensor $\mathcal{G}$ with respect to subgrid models SGS-A $(a)$, SGS-B $(b)$, SGS-C $(c)$, and SGS-D $(d)$ (see Table 1).

In Fig 8 , the $\left(R^{\mathcal{S}}, Q^{\mathcal{S}}\right)$ invariant map compares the contribution of subgrid-scale stresses to the energy cascade. The invariant, $Q^{\mathcal{S}}=-(1 / 4) \varepsilon / v$, represents the viscous dissipation $\varepsilon$ [8]. Large eddies with a negative values of $Q^{\mathcal{S}}$ are contributor to dissipation. The invariant $R^{\mathcal{S}}$ is a special form of the invariant $R^{\mathcal{G}}$ when $Q^{\mathcal{G}}<-3 \sqrt[3]{\left(R^{\mathcal{G}}\right)^{2} / 4}$. For an incompressible flow, the eigenvalues of strain tensor $\mathcal{S}$ satisfy $\lambda_{1} \geq \lambda_{2} \geq \lambda_{3}$ and $\lambda_{1}+\lambda_{2}+\lambda_{3}=0$. Thus, we can write $R^{\mathcal{S}}=-\lambda_{1} \lambda_{2} \lambda_{3}$. Similar to Eq (10), we have the following evolution equation

$$
\frac{D}{D t}\left(\frac{1}{2} \mathcal{S}_{i j} \mathcal{S}_{i j}\right)=-\mathcal{S}_{i j} \mathcal{S}_{j k} \mathcal{S}_{k i}-\frac{1}{4} \omega_{i} \omega_{j} \mathcal{S}_{i j}-\mathcal{S}_{i j} \frac{\partial^{2} P}{\partial x_{i} \partial x_{j}}+v \mathcal{S}_{i j} \nabla^{2} \mathcal{S}_{i j}
$$

The main message from Eq (11) and Fig 8 that positive values of $R^{\mathcal{S}}$ or $\lambda_{1} \lambda_{2} \lambda_{3}<0$ corresponds production of $\mathcal{S}_{i j} \mathcal{S}_{i j}$ by the strain self-amplification process. Thus, $\mathcal{R}^{\mathcal{S}}>0$ also implies sheet-like structures due to viscous dissipation with $\lambda_{1}, \lambda_{2}>0$ and $\lambda_{3}<0$. On the other hand, destruction of $\mathcal{S}_{i j} \mathcal{S}_{i j}$ is associated with tube like structures due to $\lambda_{1}>0$ and $\lambda_{2}, \lambda_{3}<0$. 

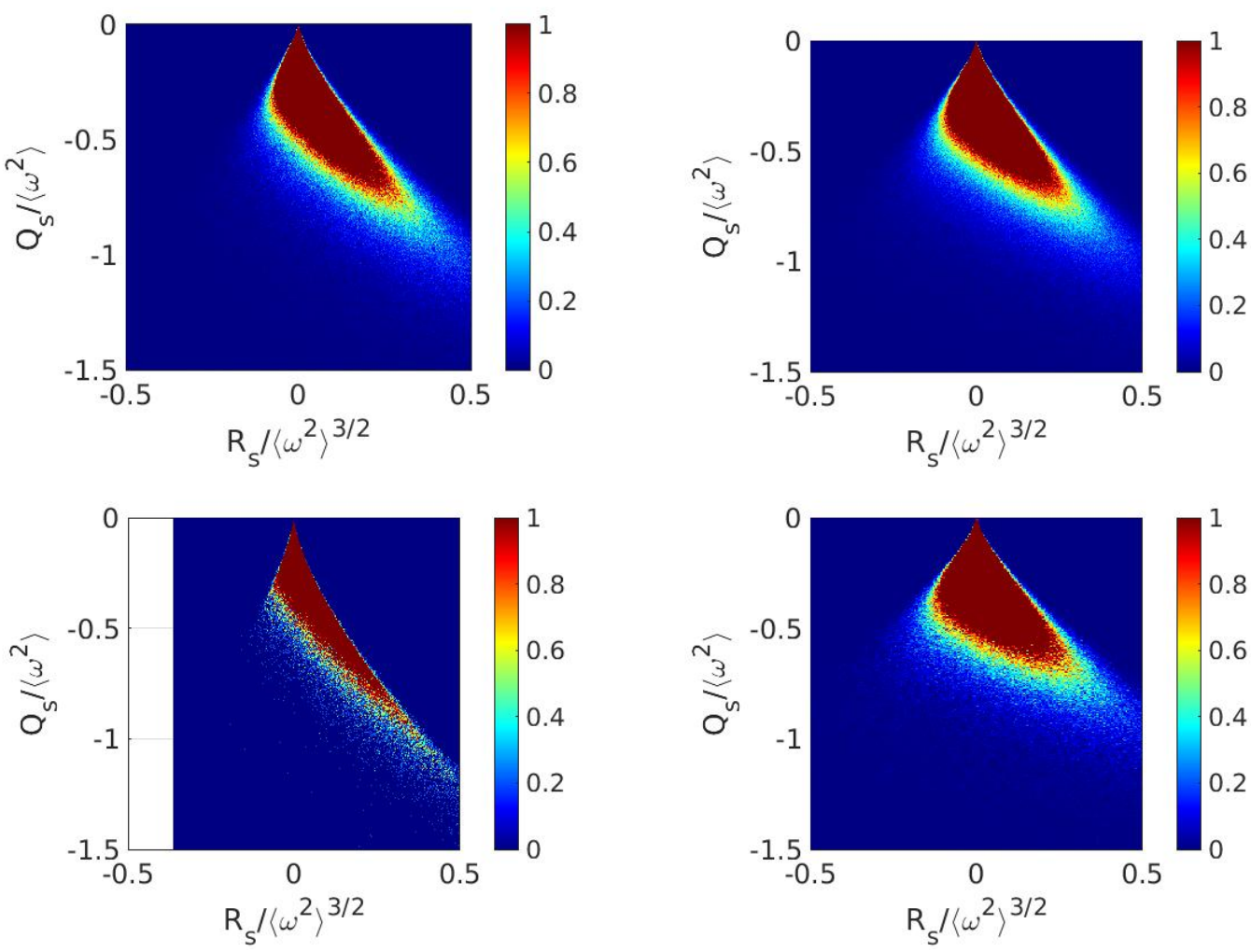

Figure 8. Plots of the joint probability density function of two invariants $R_{S}$ and $Q_{S}$ of the strain rate tensor $\mathcal{S}$.

In accordance with [45] and other investigations, strain itself is induced by vorticity because there is no other way of production of enstrophy other than straining of weak vorticity. This mechanism is called self-amplification of velocity gradient tensor. In order to link this mechanism to the energy cascade, we focus on the statistics of the rate of vortex stretching

$$
\Sigma_{\omega}=\frac{\omega_{i} \omega_{j} \mathcal{S}_{i j}}{|\boldsymbol{\omega}|}
$$

and the second invariant of the rotation-rate tensor $Q^{\mathcal{R}}$. The statistics of these quantities appear directly in the vorticity equation

$$
\frac{D \frac{1}{2}|\boldsymbol{\omega}|^{2}}{D t}=\omega_{i} \omega_{j} \mathcal{S}_{i j}+v \omega_{i} \nabla^{2} \omega_{i}
$$

Fig 9 shows some common features of the self-amplification of velocity derivatives. More specifically, the joint PDFs of the rate of vortex stretching $\Sigma_{\omega}$ with $Q^{\mathcal{R}}$ show that the highest values of the enstrophy are associated with positive but low values of $\Sigma_{\omega}$. High rates of vortex stretching and compression correlate with low values of enstrophy. The tilt towards positive values of $\Sigma_{\omega}$ implies that vortex tubes are being more stretched than compressed. Also, most of the volume in the flow is occupied by relatively 'weak' vorticity, whereas strong vortices filling only a small fraction of the space. 

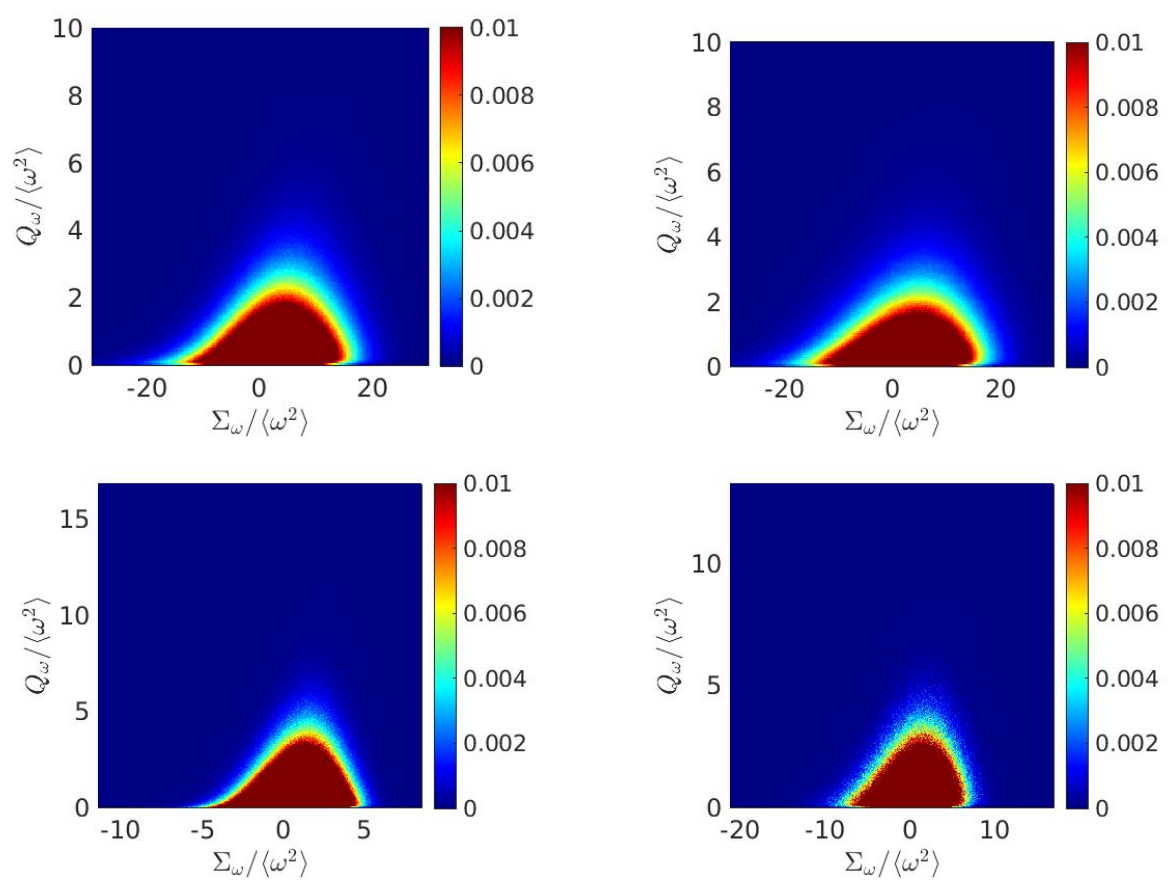

Figure 9. The plots of the joint probability density function of the second invariant $Q^{S}$ and $Q_{R}$ of two tensors $\mathcal{S}$ and $\mathcal{R}$, respectively.

Until now, we have discussed some statistical approaches to investigate the sensitivity of subgrid models in the context of vortex stretching by energy cascade. 
3.3.4. Wind turbine in the atmospheric boundary layer
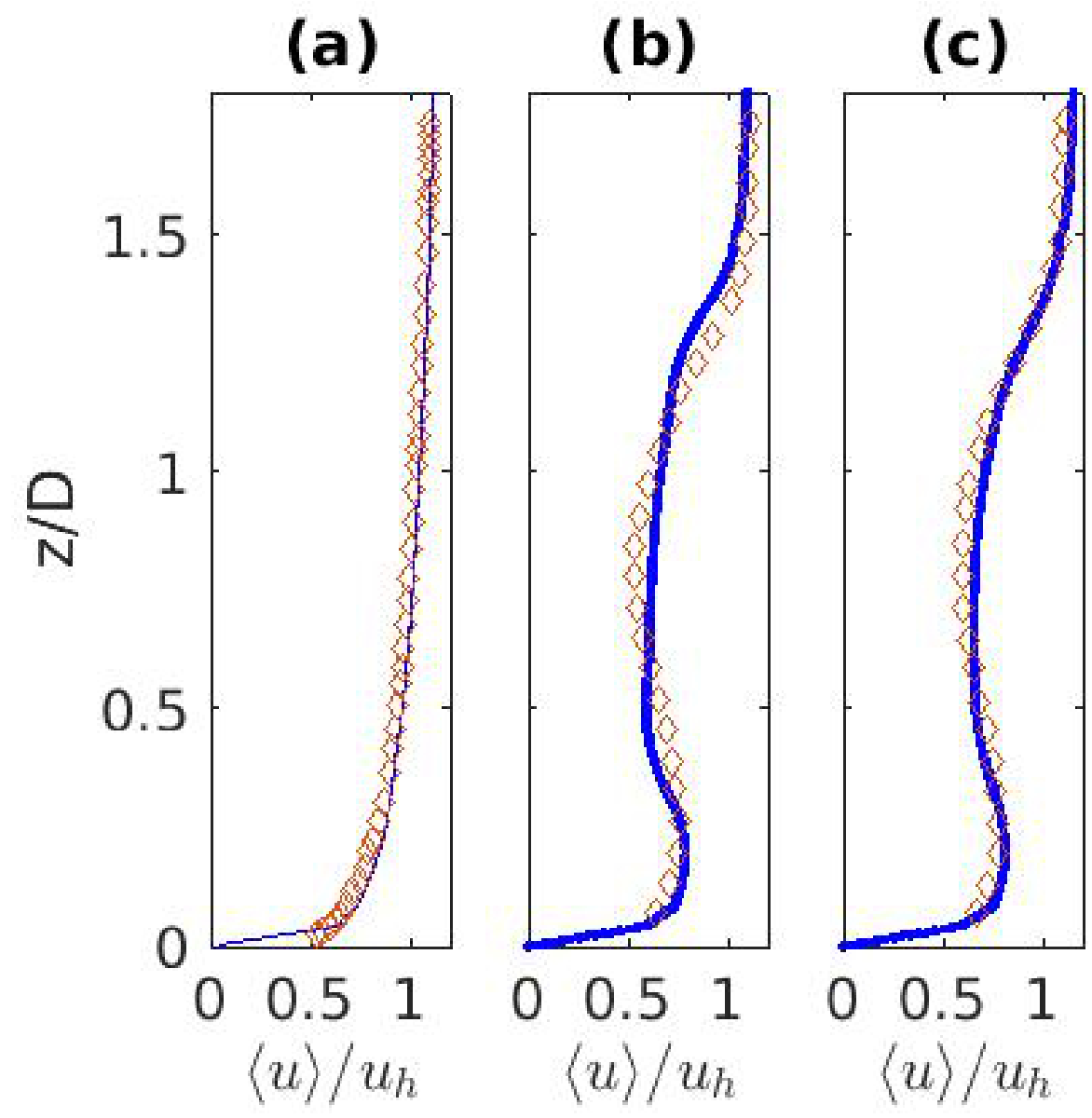

Figure 10. Comparison of the LES results using subgrid model SGS-A and the wind tunnel measurements of the stream-wise velocity $\langle\bar{U}\rangle(z)$ for atmospheric boundary layer flow over a single turbine. Wind profiles $\langle\bar{U}\rangle(z)$ are shown at $(a)$ inflow boundary, $(b)$ at a distance of $2 D$ from the wind turbine, and $c$ ) at a distance of $3 D$ from the wind turbine.

In wind engineering applications, it is desired that by setting appropriate profiles for wind velocity at the inlet, subject to other boundary conditions, the boundary layer will be maintained up to the obstructions in the flow. In this section we validate our LES code for the simulation of atmospheric boundary layer flow over a single wind turbine. Near the Earth's surface, anisotropy in turbulence structure increases, and the characteristic length-scale of the flow structures diminishes rapidly. To reproduce the wind profile, the filter width $\Delta_{\text {les }}$ is usually decreased as the wall-normal coordinate decreases, i.e. $z \rightarrow 0$. A goal of the subgrid model SGS-A, Eq (9), is to dynamically adjust the stress $\tau_{i j}$ without adjusting $\Delta_{\text {les }}$ in Eq (9). To confirm such a property of SGS-A, we have compared the result of LES with that of experimental measurement. In this study, we have set fixed values of $\Delta_{\text {les }}=2\left(\Delta_{x} \Delta_{y} \Delta_{z}\right)^{1 / 3}$, and $c_{k}=0.325$ in Eq (9).

We consider a computational domain size of $36 D \times 6 D \times 3 D$ in the stream-wise, spanwise, and vertical directions, respectively. We have chosen the domain and the number of grid points $256 \times 32 \times 72$ similar to the simulation reported by Stevens et al. [46]. At the inflow boundary, we have used $\bar{u}_{1}(z)=\left(u_{*} / \kappa\right) \log \left(z / z_{0}\right)$ for the stream-wise component of the velocity, where values of $u_{*}$ and $\kappa$ were chosen to match the experimental data. To assign transient values to the span-wise and surface normal components of the inflow velocity, we have applied a synthetic eddy generation approach. At the lower boundary $z=0$, we have applied the shear stress experienced by a rough surface of the Earth [17]. 
The upstream velocity $U_{\infty}$ is related to the local velocity $\bar{u}_{i}^{d}$ at the actuator disk by $U_{\infty}=\bar{u}_{1}^{d} /(1-a)$, where $a$ is the axial induction factor, and $d$ indicates that $\bar{u}_{i}^{d}$ is zero on all grid points outside the actuator disk. Instead of a disk-average velocity, we have represented the instantaneous thrust force experienced by each grid point $(x, y, z)$ on the actuator disk by

$$
F_{1}(x, y, z, t)=-(\rho / 2) C_{t} \sqrt{\bar{u}_{i}^{d} \bar{u}_{i}^{d}} \bar{u}_{1}^{d} D^{3} \pi / 4,
$$

where the thrust coefficient is $C_{t}=0.8248$, and rotor diameter is $D=0.15 \mathrm{~m}$ [46].

Fig 10 compares the time-averaged stream-wise velocity $\left\langle\bar{u}_{1}\right\rangle(z)$ as a function of the vertical coordinate $z$. We see that the near-turbine dynamics at the wake behind a single turbine is captured with our LES code. The accuracy of our LES code, particularly in the prediction of mean flow, is similar to that of the LES employed by Stevens et al. [46]. The most important findings include the verification of the idea that stretching of vortex tube plays a major important role in turbulence energy cascade, and that this hypothesis has the potential to accurately model atmospheric boundary layer flow over wind turbines.

\section{Conclusion and future direction}

Over the past half a century, the performance of subgrid models in LES has been widely used by scientists and engineers. It has been 25 years since Meneveau [13] introduced some statistical characteristics of subgrid models, which are often considered in either the formulation or the evaluation of subgrid models. In this article, we have compared statistics of velocity gradient tensor among four subgrid models using the decay of fully developed turbulence. However, the main focus is on understanding whether the strain self-amplification is driven by vortex stretching. Instead of highlighting the crucial role of strain-rate self-amplification in the energy cascade, which questions the role of of vorticity stretching to drive the cascade, we have computed the rate of dissipation of the subgridscale energy by four methods.

Two-point statistics, such as velocity increments and structure functions are widely used in the study of turbulent flows. In LES, filtered velocity gradients are natural candidate to encompass the information contained in two-point statistics, which is an important tool of increasing practical importance in the age of supercomputing. Our study attempts to encompass such statistical features in the dynamic procedure of subgrid models. It has been observed that, models that incorporate nonlocal and genetic effects, via two-level filtering (e.g. SGS-B), seem to reproduce statistical features similar to models (e.g. SGS-A) that directly account for the dynamics of the velocity gradient tensor. This also suggests that the global and localized dynamic models aim to account for the nonlocal rule of vortex stretching. It may help further by noting a subtle difference between vortex stretching and vorticity stretching. A further investigation of how regions of strong vorticity are often stretched along regions of strong strain might help understand how stretching of a vortex may interact with the strain self-amplification.

Considering the square of the velocity gradient tensor to define the eddy viscosity is equivalent to considering the diffusion of the vorticity, which was noted by Taylor [21] in 1932. An advantage of considering vortex stretching is that if the motion is locally limited to two dimensions, the pressure does not affect the vorticity. Vortex tubes stir the surrounding fluid to enhance turbulence mixing and transfer energy from large- to small-scales. The work of Smagorinsky [6] relies on the heuristics that pressure do not affect the mean transfer of momentum of individual eddies, or such a local effect is negligible.

It is worth mentioning that we have primarily analyzed homogeneous isotropic turbulence in the present article. First, it is necessary to identify a minimal set of a priori characteristics that are important for the study of complex flows. Second, we need to acquire an adequate computational framework to inform developments of new computational investigations that would greatly facilitate the study of more complex flows. The modeling and analysis tools discussed in this research can be applied to help develop a priori conditions in order to address comprehensive challenges of turbulence. Machine learning 
techniques are increasing attracting the fluid dynamics research community. Successful application of such techniques in the study of turbulent flows require that appropriate dynamical and statistical considerations be identified in training data.

Vortex stretching are the best candidates for achieving more accurate statistics, which can be explored in further studies of more complex geometries. LES of flow around complex geometries can be either wall-resolved (with a fine grid) or wall-modeled (with a model of shear stress). Future investigations may also include the study of wall-models for LES of complex flows, where vortex stretching accounts for the dynamics in the boundary layer adjacent to the wall-layer.

Author Contributions: "Conceptualization and methodology, J. A. and A. V.; software, J.A.; validation, M.H.; writing—original draft preparation, M. H.; writing—review and editing, J.A.; All authors have read and agreed to the published version of the manuscript."

Funding: "This research was funded by NSERC discovery grant number [to be done]." and "The APC was funded by [to be done]".

Acknowledgments: Authors acknowledge help from Mr. Jagdeep Singh in collecting the experimental data.

Conflicts of Interest: "The authors declare no conflict of interest."

\section{Abbreviations}

The following abbreviations are used in this manuscript:

CFD Computational Fluid Dynamics

LES Large Eddy Simulation

\section{References}

1. Bradshaw, P. Understanding and prediction of turbulent flow-1996. International journal of heat and fluid flow 1997, 18, 45-54.

2. Davidson, P. Turbulence: an introduction for scientists and engineers, 2004.

3. Moser, R.D.; Haering, S.W.; Yalla, G.R. Statistical Properties of Subgrid-Scale Turbulence Models. Annual Review of Fluid Mechanics 2021, 53, 255-286.

4. Pope, S.B. Turbulent flows, 2001.

5. Meneveau, C.; Lund, T.S.; Cabot, W.H. A Lagrangian dynamic subgrid-scale model of turbulence. Journal of fluid mechanics 1996, 319, 353-385.

6. Smagorinsky, J. General circulation experiments with the primitive equations: I. The basic experiment. Monthly weather review 1963, 91, 99-164.

7. Chung, D.; Pullin, D.I. Large-eddy simulation and wall modelling of turbulent channel flow. Journal of Fluid Mechanics 2009, 631, 281-309.

8. Trias, F; Folch, D.; Gorobets, A.; Oliva, A. Building proper invariants for eddy-viscosity subgrid-scale models. Physics of Fluids 2015, 27, 065103.

9. Bose, S.T.; Park, G.I. Wall-Modeled Large-Eddy Simulation for Complex Turbulent Flows. Annual Review of Fluid Mechanics 2018, 50, 535-561.

10. Eyink, G.L. Multi-scale gradient expansion of the turbulent stress tensor. Journal of Fluid Mechanics 2006, 549, 159.

11. Tsinober, A. An informal introduction to turbulence; Vol. 63, Springer Science \& Business Media, 2001.

12. Sagaut, P.; Cambon, C. Homogeneous turbulence dynamics; Cambridge University Press, 2018.

13. Meneveau, C. Statistics of turbulence subgrid-scale stresses: Necessary conditions and experimental tests. Physics of Fluids 1994, 6, 815-833.

14. Farge, M.; Schneider, K. Coherent Vortex Simulation (CVS), A Semi-Deterministic Turbulence Model Using Wavelets. Flow, Turbulence and Combustion 2001, 66, 393-426.

15. Doan, N.A.K.; Swaminathan, N.; Davidson, P.A.; Tanahashi, M. Scale locality of the energy cascade using real space quantities. Phys. Rev. Fluids 2018, 3, 084601.

16. Carbone, M.; Bragg, A.D. Is vortex stretching the main cause of the turbulent energy cascade? Journal of Fluid Mechanics 2020, 883.

17. Bhuiyan, M.A.S.; Alam, J.M. Scale-adaptive turbulence modeling for LES over complex terrain. Engineering with Computers 2020, pp. $1-13$.

18. Alam, J.M.; Fitzpatrick, L.P. Large eddy simulation of flow through a periodic array of urban-like obstacles using a canopy stress method. Computers \& Fluids 2018, 171, 65-78. 
19. Characterizing Impacts of Atmospheric Turbulence on Wind Farms Through Large Eddy Simulation (LES), Vol. Volume 10: Ocean Renewable Energy, International Conference on Offshore Mechanics and Arctic Engineering, 2019.

20. Borue, V.; Orszag, S.A. Local energy flux and subgrid-scale statistics in three-dimensional turbulence. Journal of Fluid Mechanics 1998, 366, 1-31.

21. Taylor, G.I. The transport of vorticity and heat through fluids in turbulent motion. Proceedings of the Royal Society of London. Series A, Containing Papers of a Mathematical and Physical Character 1932, 135, 685-702.

22. Taylor, G.I. Production and dissipation of vorticity in a turbulent fluid. Proceedings of the Royal Society of London. Series A-Mathematical and Physical Sciences 1938, 164, 15-23.

23. Sagaut, P.; Cambon, C. Homogeneous turbulence dynamics; Vol. 10, Springer, 2008.

24. Onsager, L. Statistical hydrodynamics. Il Nuovo Cimento (1943-1954) 1949, 6, 279-287.

25. Leonard, A.; Peters, N. Theodore von Kármán. This page intentionally left blank 2011, p. 101.

26. Bernard, P.S.; Thangam, S.; Speziale, C.G. The role of vortex stretching in turbulence modeling. In Instability, Transition, and Turbulence; Springer, 1992; pp. 563-574.

27. Kundu, P.K.; Cohen, I.M.; Dowling, D. Fluid Mechanics 4th, 2008.

28. Tennekes, H.; Lumley, J.L. A first course in turbulence; MIT press, 2018.

29. Betchov, R. An inequality concerning the production of vorticity in isotropic turbulence. Journal of Fluid Mechanics 1956, 1, 497-504.

30. Shetty, D.A.; Frankel, S.H. Assessment of stretched vortex subgrid-scale models for LES of incompressible inhomogeneous turbulent flow. International journal for numerical methods in fluids 2013, 73, 152-171.

31. Nicoud, F.; Ducros, F. Subgrid-scale stress modelling based on the square of the velocity gradient tensor. Flow, turbulence and Combustion 1999, 62, 183-200.

32. Martın, J.; Ooi, A.; Chong, M.S.; Soria, J. Dynamics of the velocity gradient tensor invariants in isotropic turbulence. Physics of Fluids 1998, 10, 2336-2346.

33. da Silva, C.B.; Pereira, J.C. Invariants of the velocity-gradient, rate-of-strain, and rate-of-rotation tensors across the turbulent/nonturbulent interface in jets. Physics of fluids 2008, 20, 055101.

34. Lund, T.S.; Novikov, E. Parameterization of subgrid-scale stress by the velocity gradient tensor. Annual Research Briefs 1992, 1992, 27-43.

35. Germano, M.; Piomelli, U.; Moin, P.; Cabot, W.H. A dynamic subgrid-scale eddy viscosity model. Physics of Fluids A: Fluid Dynamics 1991, 3, 1760-1765.

36. Deardorff, J.W.; others. A numerical study of three-dimensional turbulent channel flow at large Reynolds numbers. J. Fluid Mech 1970, 41, 453-480.

37. Deardorff, J.W. The Use of Subgrid Transport Equations in a Three-Dimensional Model of Atmospheric Turbulence. Journal of Fluids Engineering 1973, 95, 429-438.

38. Deardorff, J.W. Stratocumulus-capped mixed layers derived from a three-dimensional model. Boundary-Layer Meteorology 1980, 18, 495-527.

39. Stoll, R.; Gibbs, J.A.; Salesky, S.T.; Anderson, W.; Calaf, M. Large-Eddy Simulation of the Atmospheric Boundary Layer. Boundary-Layer Meteorology 2020, 177, 541-581.

40. Yoshizawa, A. Statistical theory for compressible turbulent shear flows, with the application to subgrid modeling. The Physics of Fluids 1986, 29, 2152-2164.

41. Piomelli, U.; Balaras, E. Wall-Layer Models For Large-Eddy Simulations. Annual Review of Fluid Mechanics 2002, 34, 349-374.

42. BARDINA, J.; FERZIGER, J.; REYNOLDS, W. Improved subgrid-scale models for large-eddy simulation. 13th Fluid and PlasmaDynamics Conference, 1980.

43. Liu, S.; Meneveau, C.; Katz, J. On the properties of similarity subgrid-scale models as deduced from measurements in a turbulent jet. Journal of Fluid Mechanics 1994, 275, 83-119.

44. Kolmogorov, A.N. A refinement of previous hypotheses concerning the local structure of turbulence in a viscous incompressible fluid at high Reynolds number. Journal of Fluid Mechanics 1962, 13, 82-85. doi:10.1017/S0022112062000518.

45. Jiménez, J.; Wray, A.A.; Saffman, P.G.; Rogallo, R.S. The structure of intense vorticity in isotropic turbulence. Journal of Fluid Mechanics 1993, 255, 65-90.

46. Stevens, R.J.; Mart́nez-Tossas, L.A.; Meneveau, C. Comparison of wind farm large eddy simulations using actuator disk and actuator line models with wind tunnel experiments. Renewable Energy 2018, 116, 470 - 478. 